\title{
On the Stability Problem of Equilibrium Discrete Planar Curves
}

\author{
Yoshiki Jikumaru' ${ }^{1}$
}

Received: 16 April 2020 / Revised: 6 November 2020 / Accepted: 22 April 2021 / Published online: 7 June 2021

(C) The Author(s) 2021

\begin{abstract}
We study planar polygonal curves with the variational methods. We show a unified interpretation of discrete curvatures and the Steiner-type formula by extracting the notion of the discrete curvature vector from the first variation of the length functional. Moreover, we determine the equilibrium curves for the length functional under the area-constraint condition and study their stability.
\end{abstract}

Keywords Discrete planar curve $\cdot$ Variational problem $\cdot$ Discrete curvature $\cdot$ Steiner formula $\cdot$ Stability

Mathematics Subject Classification 49Q10 $\cdot 53 \mathrm{~A} 04 \cdot 53 \mathrm{~A} 70 \cdot 53 \mathrm{C} 42$

\section{Introduction}

Discrete differential geometry is an active research field with connections to the smooth manifold theory and visualization on the computer. In this field, from the theoretical point of view, one approach is based on the variational problems and the other on integrability of equations. We take the former approach and focus on discrete planar curves. Surprisingly, such a discrete curve theory is not well understood, therefore we further develop it inspired by the work [10]. Moreover, we consider the stability problem for equilibrium discrete curves. The stability problem for discrete objects has not developed well after the work by Polthier and Rossman [11].

First we derive the first variation formula and extract a vector from the formula, called the discrete curvature vector in Sect. 2. In Sect. 3, by using this curvature vector,

Editor in Charge: János Pach

Yoshiki Jikumaru

y-jikumaru@imi.kyushu-u.ac.jp

1 Institute of Mathematics for Industry, Kyushu University, 744 Motooka Nishi-ku, Fukuoka 819-0395, Japan 
we show a unified interpretation which derives various kinds of discrete curvature notions introduced in $[7,8]$. The important viewpoint here is that there is no natural notion of the line element on the vertices. In Sect. 4, we characterize equilibrium curves for the length functional under the area-constraint condition as regular polygons. In Sect. 5, we derive a Steiner-type formula for parallel curves by using the "vertex normal" constructed from the discrete curvature vector derived in Sect. 2. In Sect. 6, we consider the stability problem of the regular polygons. We can derive the second variation formula, similar to the smooth case, by decomposing the variation vector field into the "normal" and "tangential" directions as in [1]. Moreover, we show the instability for the non-convex regular polygons by using the second variation formula for "normal" variations in Sect. 7.

\section{The First Variation Formula}

In this section we will consider the variation of discrete curves and extract the "curvature vector" from the first variation formula. We can expect that this vector gives a notion of the curvature and normal at vertices. Let us recall the basic definition of discrete planar curves.

Definition 2.1 Let $n$ be a non-negative integer. A standard (abstract) $n$-path is a simplicial complex $G=(V, E)$ formed by

- $n+1$ abstract points $V=\left\{v_{0}, \ldots, v_{n}\right\}$,

- the set of $n$ edges $e_{k}=\left[v_{k}, v_{k+1}\right], k=0, \ldots, n-1$.

A standard abstract $n$-circle is the union of a standard $n$-path and the "final" edge $e_{n}=\left[v_{n}, v_{0}\right]$. A discrete (planar) curve is a geometric realization of a standard $(n-1)$ path or $(n-1)$-circle, which is a map $X: V \rightarrow \mathbb{R}^{2}$ satisfying $l_{k}:=\left|p_{k+1}-p_{k}\right| \neq 0$ for all $k=0,1, \ldots, n-1$, where we denote $p_{k}:=X\left(v_{k}\right)$. We denote such a discrete curve as $\Gamma_{h}=\left\{p_{k}\right\}_{k}$.

To each oriented edge $e_{k}:=\left[p_{k}, p_{k+1}\right]$ of $\Gamma_{h}$ we can assign a unit normal vector

$$
v_{k}:=R\left(\frac{p_{k+1}-p_{k}}{l_{k}}\right)=R\left(\frac{p_{k+1}-p_{k}}{\left|p_{k+1}-p_{k}\right|}\right), \quad k=0, \ldots, n-1,
$$

where $R$ is the $(\pi / 2)$-rotation or $(-\pi / 2)$-rotation in $\mathbb{R}^{2}$. It does not matter which one we choose, but we choose the same $R$ for all $k$. For a discrete curve $\Gamma_{h}=\left\{p_{k}\right\}_{k}$ with an orientation, the length of $\Gamma_{h}$ and the 2-dimensional volume (area) bounded by $\Gamma_{h}$ are defined by

$$
L\left(\Gamma_{h}\right):=\sum_{k} l_{k}=\sum_{k}\left|p_{k+1}-p_{k}\right|, \quad \text { Area } \Gamma_{h}:=\frac{1}{2} \sum_{k}\left\langle p_{k}, v_{k}\right\rangle\left|p_{k+1}-p_{k}\right| .
$$

In these settings, we consider the following question:

What are the unit normal, curvature, and line element at the vertices? 
A realization of the dual graph may give an answer for this question, but we do not consider such a realization. In order to approach the problem, we will extract the discrete curvature vector from the first variation of the length. Therefore we first derive the first variation formula of the length functional. In the discrete setting we consider the variation of vertices, that is, piecewise linear variations. We consider a variation

$$
p_{k}(t)=p_{k}+t v_{k}+O\left(t^{2}\right), \quad k=0, \ldots, n-1,
$$

where ${ }^{t} \vec{v}=\left({ }^{t} v_{0}, \ldots,{ }^{t} v_{n-1}\right) \in \mathbb{R}^{2 n}$ is the "variation vector field". If $p_{k}$ is a boundary point of $\Gamma_{h}$, then we assume $v_{k}=0$.

We want to find the vector ${ }^{t} \nabla L \in \mathbb{R}^{2 n}$ satisfying

$$
\delta L:=\left.\frac{d L}{d t}\right|_{t=0}=\langle\vec{v}, \nabla L\rangle_{\mathbb{R}^{2 n}}=\sum_{k}\left\langle v_{k}, \nabla_{p_{k}} L\right\rangle_{\mathbb{R}^{2}},
$$

where we write ${ }^{t} \nabla L=\left({ }^{t} \nabla_{p_{1}} L, \ldots,{ }^{t} \nabla_{p_{n}} L\right) \in \mathbb{R}^{2 n}$. Since this is just a directional derivative in $\mathbb{R}^{2 n}$, the following proposition is immediate:

Proposition 2.2 Let $\Gamma_{h}=\left\{p_{k}\right\}_{k}$ be a discrete closed curve and $p_{k}$ an interior vertex. Then the gradient of the length can be expressed as in the following formula:

$$
\nabla_{p_{k}} L=R\left(v_{k}-v_{k-1}\right)=-\frac{p_{k+1}-p_{k}}{l_{k}}+\frac{p_{k}-p_{k-1}}{l_{k-1}} .
$$

By using this formula we have

$$
\delta L=\sum_{k}\left\langle\nabla_{p_{k}} L, v_{k}\right\rangle=-\sum_{k}\left\langle-\frac{1}{L_{k}} \nabla_{p_{k}} L, v_{k}\right\rangle L_{k},
$$

where we inserted some auxiliary function $L_{k}$ defined on the vertices. This kind of observation is essentially remarked on in the paper [3].

Definition 2.3 (discrete curvature vector) For a positive function $L_{k}$ defined on the vertices, we call the vector

$$
\tilde{N}_{k}=-\frac{1}{L_{k}} \nabla_{p_{k}} L=\frac{1}{L_{k}}\left(\frac{p_{k+1}-p_{k}}{l_{k}}-\frac{p_{k}-p_{k-1}}{l_{k-1}}\right)
$$

the discrete curvature vector with respect to $L_{k}$.

Remark 2.4 There are some reasons why we can regard the vector $\widetilde{N}_{k}$ as the curvature vector. First, the vector $\widetilde{N}_{k}$ is independent of the choice of the unit normal, i.e., an intrinsic quantity. Moreover, the second expression (2.2) can be regarded as a discretization of a part of the Frenet-Serret formula. As we will remark below, suitable choices of the function $L_{k}$ derive various notions of discrete curvature defined in [7,8]. Non-uniqueness of the function $L_{k}$ comes from the fact that there is no natural line element, i.e., the metric, at vertices. 


\section{Relation with Other Notions of the Discrete Curvature}

In this section, non-uniqueness of the line element at the vertices gives various notions of the discrete curvature defined in $[7,8]$.

To describe the curvature notions, we have to define the angles at vertices. We define (the absolute value of) the angle between $v_{k-1}$ and $v_{k}$ as $\theta_{k}$, i.e.,

$$
\cos \theta_{k}:=\left\langle v_{k}, v_{k-1}\right\rangle=\frac{\left\langle p_{k+1}-p_{k}, p_{k}-p_{k-1}\right\rangle}{\left|p_{k+1}-p_{k}\right| \cdot\left|p_{k}-p_{k-1}\right|} .
$$

We have to care about the signature of $\theta_{k}$. Let $R_{\theta}$ be the $\theta$-rotation in $\mathbb{R}^{2}$. We assign the signature depending on the choice of the rotation $R$ appearing in the definition of the edge normal:

$$
\sigma:= \begin{cases}+1 & \text { if } R=R_{\pi / 2} \\ -1 & \text { if } R=R_{-\pi / 2}\end{cases}
$$

In this situation the signature of $\theta_{k}$ is determined by the equation $R_{\sigma} \theta_{k}\left(v_{k-1}\right)=v_{k}$. We easily see that $\sum_{k} \theta_{k}=2 m \pi$ for some integer $m \in \mathbb{Z}$ for any closed curve. We define the discrete curvature with respect to the choice of $L_{k}$ by the length of the discrete curvature vector with respect to $L_{k}$ :

Definition 3.1 For a positive function $L_{k}$ defined on the vertices, we call the value

$$
\kappa\left(p_{k}\right):=\frac{2 \sin \left(\theta_{k} / 2\right)}{L_{k}}
$$

the discrete curvature with respect to $L_{k}$.

Remark 3.2 If we use the expression $\nabla_{p_{k}} L=R\left(v_{k}-v_{k-1}\right)$, the discrete curvature with respect to $L_{k}$ can be written as $\kappa\left(p_{k}\right)=\left|v_{k}-v_{k-1}\right| / L_{k}$ up to the signature. This can be regarded as a discretization of the curvature for a regular planar curve.

In the lecture notes by Tim Hoffmann [8], three kinds of notions of the curvature for discrete curves are introduced:

- the curvature at vertices by using the vertex osculating circle method,

- the curvature at edges by using the edge osculating circle method, and

- the curvature at vertices by using edge osculating circle for "arclength parametrized" curve.

Moreover, Hatakeyama [7] also defined the curvature for discrete curves another way. We will show that these curvature notions can be derived from our viewpoint.

Proposition 3.3 (vertex osculating circle method [8]) If we choose

$$
L_{k}=\frac{\left|p_{k+1}-p_{k-1}\right|}{2 \cos \left(\theta_{k} / 2\right)}=\frac{\left|p_{k+1}-p_{k}+p_{k}-p_{k-1}\right|}{2 \cos \left(\theta_{k} / 2\right)}
$$


then the discrete curvature with respect to $L_{k}$ becomes

$$
\kappa\left(p_{k}\right)=\frac{2 \sin \theta_{k}}{\left|p_{k+1}-p_{k-1}\right|}=\frac{2 \sin \theta_{k}}{\left|p_{k+1}-p_{k}+p_{k}-p_{k-1}\right|}
$$

and this value coincides with the curvature based on the vertex osculating circle method.

Proposition 3.4 (for the arclength parametrized curves [8]) Assume $l_{k}=l_{k-1}=l_{0}$. If we choose

$$
L_{k}=l_{0} \cos \frac{\theta_{k}}{2}=\frac{l_{k}+l_{k-1}}{2} \cdot \cos \frac{\theta_{k}}{2},
$$

then the discrete curvature with respect to $L_{k}$ becomes

$$
\kappa\left(p_{k}\right)=\frac{2}{l_{0}} \tan \frac{\theta_{k}}{2}
$$

and this value coincides with the curvature of arclength parametrized curve.

In the paper [7], the discrete curvature at a vertex is defined as

$$
\kappa\left(p_{k}\right):=\frac{1}{\left|p_{k}-p_{k-1}\right|} \cdot\left|\frac{p_{k+1}-p_{k}}{\left|p_{k+1}-p_{k}\right|}-\frac{p_{k}-p_{k-1}}{\left|p_{k}-p_{k-1}\right|}\right|=-\frac{\left|\nabla_{p_{k}} L\right|}{\left|p_{k}-p_{k-1}\right|} .
$$

Then we immediately have the following result:

Proposition 3.5 If we choose $L_{k}=l_{k-1}=\left|p_{k}-p_{k-1}\right|$, then the discrete curvature with respect to $L_{k}$ coincides with the discrete curvature defined in [7].

Before considering the edge osculating circle method, we shall modify the first variation formula from the vertex-based expression to the edge-based expression. If we put $v_{k}=\left(w_{k}+w_{k-1}\right) / 2$, then we have

$$
\begin{aligned}
\delta L & =\frac{1}{2} \sum_{k}\left\langle\nabla_{p_{k}} L, w_{k}+w_{k-1}\right\rangle \\
& =\frac{1}{2} \sum_{k}\left\langle\nabla_{p_{k}} L+\nabla_{p_{k+1}} L, w_{k}\right\rangle=-\sum_{k}\left\langle\frac{R\left(v_{k-1}-v_{k+1}\right)}{2 L_{k}^{\prime}}, w_{k}\right\rangle L_{k}^{\prime},
\end{aligned}
$$

where $L_{k}^{\prime}$ is some auxiliary function. As in the vertex case, we call the value

$$
\kappa\left(e_{k}\right):=\frac{1}{L_{k}^{\prime}} \cdot \sin \frac{\theta_{k}+\theta_{k+1}}{2}
$$

the discrete curvature at the edge $e_{k}=\left[p_{k}, p_{k+1}\right]$ with respect to $L_{k}^{\prime}$. 
Proposition 3.6 (edge osculating circle method [8]) If we choose

$$
L_{k}^{\prime}=l_{k} \cos \frac{\theta_{k}}{2} \cos \frac{\theta_{k+1}}{2}=\left|p_{k+1}-p_{k}\right| \cdot \cos \frac{\theta_{k}}{2} \cos \frac{\theta_{k+1}}{2},
$$

then the discrete curvature with respect to $L_{k}^{\prime}$ becomes

$$
\kappa\left(e_{k}\right)=\frac{\tan \left(\theta_{k} / 2\right)+\tan \left(\theta_{k+1} / 2\right)}{\left|p_{k+1}-p_{k}\right|}
$$

and this value coincides with the curvature based on the edge osculating circle method.

Remark 3.7 To define the discrete curvature, we have to choose $L_{k}$ (respectively $L_{k}^{\prime}$ ) properly. That means if $l_{k}, l_{k-1} \rightarrow d s$ and $\theta_{k} \rightarrow 0$, then $L_{k}$ (respectively $L_{k}^{\prime}$ ) must converge to $d s$, i.e., $L_{k}$ must be a "good" candidate for a discrete line element. We can check that $L_{k}$ and $L_{k}^{\prime}$ satisfy this condition in the above examples.

Remark 3.8 (relations with the discrete Laplacian) On a discrete curve $\Gamma_{h}$ with the vertex set $V$, we consider a function $\psi: V \rightarrow \mathbb{R}$. Then the gradient and the Laplacian of $\psi$ can be defined as

$$
\begin{aligned}
\nabla \psi_{k} & :=\frac{\psi_{k+1}-\psi_{k}}{l_{k}}, \\
\Delta \psi_{k} & :=\frac{1}{L_{k}}\left(\nabla \psi_{k}-\nabla \psi_{k-1}\right)=\frac{1}{L_{k}}\left(\frac{\psi_{k+1}-\psi_{k}}{l_{k}}-\frac{\psi_{k}-\psi_{k-1}}{l_{k-1}}\right),
\end{aligned}
$$

where we denote $\psi_{k}:=\psi(k)$. Since the Laplacian should be a linear operator and determined by a local property, this definition can be seen as a special case of the discrete Laplacian from [12]. Note that the gradient is an "edge-based operator" but the Laplacian is a "vertex-based" operator. In addition, the discrete curvature vector $\widetilde{N}_{k}$ with respect to $L_{k}$ can be written as $\Delta p_{k}$.

From another point of view, if we define the Dirichlet energy of $\psi$ as

$$
E_{h}(\psi):=\frac{1}{2} \sum_{k}\left|\nabla \psi_{k}\right|^{2} l_{k}=\frac{1}{2} \sum_{k} \frac{\left|\psi_{k+1}-\psi_{k}\right|^{2}}{l_{k}},
$$

then the first variation of the energy becomes

$$
\begin{aligned}
\delta E_{h}(\psi) & =\sum_{k} \frac{\left\langle\psi_{k+1}-\psi_{k}, \varphi_{k+1}-\varphi_{k}\right\rangle}{l_{k}}=\sum_{k}\left\langle\nabla \psi_{k-1}-\nabla \psi_{k}, \varphi_{k}\right\rangle \\
& =-\sum_{k}\left\langle\Delta \psi_{k}, \varphi_{k}\right\rangle L_{k},
\end{aligned}
$$

where we take the variation of $\psi$ as $\psi_{k}(t)=\psi_{k}+t \varphi_{k}+O\left(t^{2}\right)$. Therefore $\delta E_{h}(\psi)=0$ if and only if $\Delta \psi_{k}=0$. Note that the condition $\Delta \psi_{k}=0$ is independent of the choice of $L_{k}$. 
As in the curvature case, the Laplacian can be changed since there is no natural "line element divisor $L_{k}$ ". However, with another function $\varphi: V \rightarrow \mathbb{R}$, we still have the following properties, since the quantities $\Delta \psi_{k} L_{k}$ are independent of $L_{k}$ :

- If $\psi$ is constant, then $\Delta \psi=0$.

- The condition $\Delta \psi=0$ is independent of the choice of $L_{k}$ and in this case we have the mean value property:

$$
\psi_{k}=\frac{l_{k-1}}{l_{k}+l_{k-1}} \psi_{k+1}+\frac{l_{k}}{l_{k}+l_{k-1}} \psi_{k-1}
$$

- $L^{2}$ symmetric property:

$$
\sum_{k} \psi_{k} \cdot \Delta \varphi_{k} \cdot L_{k}=\sum_{k} \Delta \psi_{k} \cdot \varphi_{k} \cdot L_{k}
$$

Note that the summation is vertex-based.

- Integration by parts:

$$
-\sum_{k} \psi_{k} \cdot \Delta \varphi_{k} \cdot L_{k}=\sum_{k} \nabla \psi_{k} \cdot \nabla \varphi_{k} \cdot l_{k}
$$

Note that the right hand side is an edge-based summation but the left hand side is a vertex-based summation. As a corollary, the operator $-\Delta$ is positive semi-definite.

\section{Equilibrium Curves of the Length Functional}

In the previous section, we showed that non-uniqueness of the line element at vertices gives various discrete curvature notions. However, the equilibrium curves for the length functional under the area-constraint condition should be characterized as some "constant curvature" objects by virtue of the smooth case. In this section, we show that such equilibrium curves can be characterized as regular polygons and that they certainly can be regarded as "constant curvature" objects. By a direct calculation we have the following result.

Lemma 4.1 For any vertex $p_{k}$ of $\Gamma_{h}$ the gradient of the area, $\nabla_{p_{k}}$ Area, is given by

$$
\nabla_{p_{k}} \text { Area }=\frac{1}{2} R\left(p_{k+1}-p_{k-1}\right)
$$

Remark 4.2 We can modify the first variation formula of the volume as follows:

$$
\delta \text { Area }=\frac{1}{2} \sum_{k}\left\langle R\left(p_{k+1}-p_{k}+p_{k}-p_{k-1}\right), v_{k}\right\rangle=\sum_{k}\left\langle\frac{l_{k} v_{k}+l_{k-1} v_{k-1}}{2 L_{k}}, v_{k}\right\rangle L_{k} .
$$




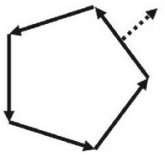

(a) $k=-1 / \cos (\pi / 5)$

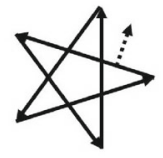

(b) $k=-1 / \cos (2 \pi / 5)$

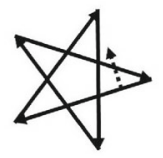

(c) $k=-1 / \cos (3 \pi / 5)$

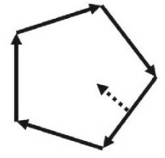

(d) $k=-1 / \cos (4 \pi / 5)$

Fig. 1 Convex and non-convex regular 5-gons with radii $a=1$

It is natural to choose $2 L_{k}=l_{k}+l_{k-1}=\operatorname{Length}(\star(p))$ and this is also frequently used as a "vertex normal" (a weighted sum of the edge normals):

$$
N_{k}^{A}:=\frac{l_{k} v_{k}+l_{k-1} v_{k-1}}{l_{k}+l_{k-1}} .
$$

In addition, we have

$$
-\nabla_{p_{k}} L=R\left(v_{k-1}-v_{k}\right)=\frac{\sin \theta_{k}}{1+\cos \theta_{k}}\left(v_{k}+v_{k-1}\right)=2 \tan \frac{\theta_{k}}{2} \cdot \frac{v_{k}+v_{k-1}}{2},
$$

by a simple calculation. Therefore, unless the curve is arclength parameterized, there are (at least) two choices of the "vertex normal" from the variational viewpoint: using the length gradient (length descent direction) or using the volume gradient (volume descent direction). This suggests that, in contrast to the smooth case, we have to choose the "preferred" vertex normal according to the energy in question.

Example 4.3 (regular polygons) Let us take a discrete curve $\Gamma_{h}^{m, n}=\left\{p_{k}\right\}_{k}$ as follows (inducing non-convex regular $n$-gon with radius $a$ ):

$$
p_{k}=a \exp \frac{2 m k \pi \sqrt{-1}}{n}, \quad k=0, \ldots, n-1, \quad m=1, \ldots, n-1,
$$

where we assume that $m / n \neq 1 / 2$. In particular, we sometimes call the curve $\Gamma_{h}^{1, n}$ a convex regular $n$-gon. Note that $\Gamma_{h}^{n-1, n}$ is also convex but it has an opposite unit normal with $\Gamma_{h}^{1, n}$ (usually we assume that $\Gamma_{h}^{1, n}$ has the outward-pointing unit normal). Then the curve $\Gamma_{h}^{m, n}$ is a critical point of the functional Length $+\kappa$. Area with $\kappa=-1 /(a \cos (m \pi / n))$. This value is the reciprocal of the radius of the inscribed circle of the polygon (up to the signature). We sometimes say that a convex regular $n$-gon with radius $a$ (and outward-pointing unit normal) has constant curvature $\kappa_{n}=$ $-1 /(a \cos (\pi / n))$. Note that $\cos (\pi m / n)=-\cos (\pi(n-m) / n)$ and $\kappa_{n} \rightarrow-1 / a$ when $n \rightarrow \infty$.

We will show that these regular polygons are the only equilibrium curves for the functional $L+\kappa \cdot$ Area.

Theorem 4.4 Let $\Gamma_{h}=\left\{p_{k}\right\}_{k=1}^{n}$ be a closed discrete curve and take $\kappa \in \mathbb{R} \backslash\{0\}$. Then the following two conditions are equivalent:

- $\Gamma_{h}$ is an equilibrium curve of the functional $L+\kappa \cdot$ Area. 
- There exist numbers $l_{0}$ and $\theta_{0}$ such that $l_{k} \equiv l_{0}, \theta_{k} \equiv \theta_{0}$, and $\kappa l_{0}=2 \tan \left(\theta_{0} / 2\right)$, i.e., $\Gamma_{h}$ must be a regular polygon.

\section{Proof We put}

$$
A_{k}:=\left(v_{k}-v_{k-1}\right)+\frac{\kappa}{2}\left(p_{k+1}-p_{k-1}\right), \quad k=1, \ldots, n .
$$

Then the discrete curve $\Gamma_{h}$ is a critical point of the functional Length $+\kappa \cdot$ Area if and only if $A_{k}=0$ for all $k$. By a simple calculation we have

$$
\begin{aligned}
\left\langle A_{k}, v_{k-1}\right\rangle & =\left(\frac{\kappa l_{k}}{2}-\tan \frac{\theta_{k}}{2}\right) \sin \theta_{k}, \\
\left\langle A_{k}, v_{k}\right\rangle & =\left(\tan \frac{\theta_{k}}{2}-\frac{\kappa l_{k-1}}{2}\right) \sin \theta_{k}, \\
\left\langle A_{k+1}, v_{k+1}\right\rangle & =\left(\tan \frac{\theta_{k+1}}{2}-\frac{\kappa l_{k}}{2}\right) \sin \theta_{k+1} .
\end{aligned}
$$

For the necessity, that is, if we assume $A_{k}=0$ for all $k$, then it follows from (4.1) and (4.2) that $\kappa l_{k}=2 \tan \left(\theta_{k} / 2\right)=\kappa l_{k-1}$. And it also follows from (4.2), (4.3), and using $l_{k}=l_{k-1}$ that

$$
\tan \frac{\theta_{k}}{2}=\frac{\kappa l_{k}}{2}=\frac{\kappa l_{k-1}}{2}=\tan \frac{\theta_{k-1}}{2}
$$

To prove the sufficiency, since $v_{k}$ and $p_{k}-p_{k-1}$ form a basis of $\mathbb{R}^{2}$ and $\left\langle A_{k}, v_{k}\right\rangle=0$ by (4.1) and the assumption, all we have to prove is $\left\langle A_{k}, p_{k}-p_{k-1}\right\rangle=0$ for all $k$. By using the assumption $l_{k}=l_{k-1}=l_{0}$ and $\theta_{k}=\theta_{0}$, we have

$$
\begin{aligned}
& \left\langle A_{k}, \frac{p_{k}-p_{k-1}}{l_{k}}\right\rangle=-\sin \theta_{k}+\frac{\kappa}{2}\left(l_{k} \cos \theta_{k}+l_{k-1}\right) \\
& \quad=\left(1+\cos \theta_{0}\right)\left(\frac{\kappa l_{0}}{2}-\frac{\sin \theta_{0}}{1+\cos \theta_{0}}\right)=\left(1+\cos \theta_{0}\right)\left(\frac{\kappa l_{0}}{2}-\tan \frac{\theta_{0}}{2}\right)=0 .
\end{aligned}
$$

This shows $A_{k}=0$ and proves the statement.

Remark 4.5 The equilibrium condition $A_{k}=v_{k}-v_{k-1}+(\kappa / 2)\left(p_{k+1}-p_{k-1}\right)=0$ is equivalent to the condition $v_{k}+(\kappa / 2)\left(p_{k+1}+p_{k}\right) \equiv c$ for some constant vector $c \in \mathbb{R}^{2}$. The latter condition can be considered as a conservation law for the EulerLagrange equation $A_{k}=0$. Since the vector $c \in \mathbb{R}^{2}$ is just a translation of the curve, we can put $c=0$ and in this case we have $\left(p_{k+1}+p_{k}\right) / 2=-v_{k} / \kappa$. Therefore, the edge midpoints must be tangent to the unit circle.

We found that equilibrium closed curves of the functional $L+\kappa \cdot$ Area must satisfy $l_{k} \equiv l_{0}$, i.e., they must have "good coordinates (arclength parameter)". If we note that we can define the curvature at vertices for an arclength parametrized curve, the previous result can be restated as follows: 
Corollary 4.6 Let $\Gamma_{h}$ be an arclength parametrized discrete closed curve, i.e., $l_{k} \equiv l_{0}$, and $\kappa \in \mathbb{R} \backslash\{0\}$. Then the following two conditions are equivalent:

- $\Gamma_{h}$ is an equilibrium curve of the functional Length $+\kappa \cdot$ Area.

- The discrete curvature $\left(2 / l_{0}\right) \tan \left(\theta_{k} / 2\right)$ is constant and $=\kappa$.

\section{Parallel Curves and Steiner-Type Formula}

In this section we will derive the discrete version of the Steiner-type formula in order to show effectiveness of the vertex normal we will define. The following type of Steiner formula has in fact appeared in some papers, e.g., [2,4]. There, however, the notion of curvature is tried to be found with the Steiner-type formula; here we will derive the Steiner-type formula by using our vertex normal and connect it with the well-known notion of curvature.

Let $\Gamma_{h}=\left\{p_{k}\right\}_{k}$ be a discrete curve and take an interior vertex $p_{k}$. We can expect that if we normalize the discrete curvature vector, then we have the "vertex normal". Recall that the length of the length gradient $\nabla_{p_{k}} L$ can be computed as $2 \sin \left(\theta_{k} / 2\right)$ up to the signature. In the discrete case, we should consider another factor $2 \sin \left(\theta_{k} / 2\right) \cos \left(\theta_{k} / 2\right)=\sin \theta_{k}$ and put

$$
N_{k}:=-\frac{\nabla_{p_{k}} L}{\sin \theta_{k}}=\frac{R\left(v_{k-1}-v_{k}\right)}{\sin \theta_{k}}=\frac{1}{1+\cos \theta_{k}}\left(v_{k}+v_{k-1}\right)
$$

Then we shall call the vector $N_{k}$ the vertex normal at $p_{k}$. The second expression in (5.1) allows us to define the vertex normal even if $\theta_{k}=0$. Then we consider the following deformation of the curve:

$$
p_{k}(t)=p_{k}+t N_{k}, \quad k=1, \ldots, n .
$$

Lemma 5.1 We have $\left\langle p_{k+1}(t)-p_{k}(t), v_{k}\right\rangle=0$; therefore we call this deformation parallel curves.

Proof. This is a direct calculation:

$$
\begin{aligned}
\left\langle p_{k+1}(t)-p_{k}(t), v_{k}\right\rangle & =\left\langle\left(p_{k+1}-p_{k}\right)+t\left(N_{k+1}-N_{k}\right), v_{k}\right\rangle \\
& =t\left(\frac{\left\langle v_{k+1}+v_{k}, v_{k}\right\rangle}{1+\cos \theta_{k+1}}-\frac{\left\langle v_{k}+v_{k-1}, v_{k}\right\rangle}{1+\cos \theta_{k}}\right) \\
& =t\left(\frac{1+\cos \theta_{k+1}}{1+\cos \theta_{k+1}}-\frac{1+\cos \theta_{k}}{1+\cos \theta_{k}}\right)=0 .
\end{aligned}
$$

Theorem 5.2 (discrete Steiner-type formula) For parallel curves $\left\{p_{k}(t)\right\}_{k}$, we have

$$
\left|p_{k+1}(t)-p_{k}(t)\right|=\left|p_{k+1}-p_{k}\right| \cdot\left(1-t \kappa\left(e_{k}\right)\right),
$$




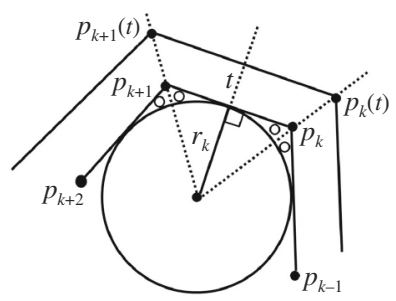

Fig. 2 Parallel curves

where $\kappa\left(e_{k}\right)$ is the discrete curvature based on the edge osculating circle method [8]:

$$
\kappa\left(e_{k}\right)=\frac{\tan \left(\theta_{k} / 2\right)+\tan \left(\theta_{k+1} / 2\right)}{\left|p_{k+1}-p_{k}\right|} .
$$

Before giving the proof, we give an intuitive explanation for a special case. In Fig. 2, the similarity ratio of the triangles gives

$$
\begin{aligned}
& \left|p_{k+1}(t)-p_{k}(t)\right|:\left|p_{k+1}-p_{k}\right|=\left(-r_{k}+t\right):-r_{k} \\
& \quad \Longleftrightarrow\left|p_{k+1}(t)-p_{k}(t)\right|=\left|p_{k+1}-p_{k}\right| \cdot\left(1-t \kappa\left(e_{k}\right)\right) .
\end{aligned}
$$

Note that the signature of the curvature radius $r_{k}$ is negative in the figure.

Proof. By direct calculation we have

$$
\begin{aligned}
&\left\langle\frac{p_{k+1}-p_{k}}{\left|p_{k+1}-p_{k}\right|}, N_{k+1}-N_{k}\right\rangle=\left\langle-R v_{k}, \frac{v_{k}+v_{k+1}}{1+\cos \theta_{k+1}}-\frac{v_{k-1}+v_{k}}{1+\cos \theta_{k}}\right\rangle \\
&=-\left(\frac{\sin \theta_{k+1}}{1+\cos \theta_{k+1}}+\frac{\sin \theta_{k}}{1+\cos \theta_{k}}\right)=-\left(\tan \frac{\theta_{k+1}}{2}+\tan \frac{\theta_{k}}{2}\right), \\
&\left|N_{k+1}-N_{k}\right|^{2}=\frac{1}{\cos ^{2}\left(\theta_{k+1} / 2\right)}+\frac{1}{\cos ^{2}\left(\theta_{k} / 2\right)}-2\left\langle N_{k}, N_{k+1}\right\rangle \\
&=2+\tan ^{2} \frac{\theta_{k+1}}{2}+\tan ^{2} \frac{\theta_{k}}{2}-2 \frac{1+\cos \theta_{k}+\cos \theta_{k+1}+\cos \left(\theta_{k}+\theta_{k+1}\right)}{\left(1+\cos \theta_{k}\right)\left(1+\cos \theta_{k+1}\right)} \\
&=\left(\tan \frac{\theta_{k}}{2}+\tan \frac{\theta_{k+1}}{2}\right)^{2} .
\end{aligned}
$$

Therefore we conclude

$$
\begin{aligned}
\mid p_{k+1}(t)- & \left.p_{k}(t)\right|^{2} \\
= & \left|p_{k+1}-p_{k}\right|^{2}+2 t\left\langle p_{k+1}-p_{k}, N_{k+1}-N_{k}\right\rangle \\
& \quad+t^{2}\left|N_{k+1}-N_{k}\right|^{2}
\end{aligned}
$$




$$
\begin{aligned}
= & \left|p_{k+1}-p_{k}\right|^{2}-2 t\left|p_{k+1}-p_{k}\right|\left(\tan \frac{\theta_{k}}{2}+\tan \frac{\theta_{k+1}}{2}\right) \\
& +t^{2}\left(\tan \frac{\theta_{k}}{2}+\tan \frac{\theta_{k+1}}{2}\right)^{2} \\
= & \left|p_{k+1}-p_{k}\right|^{2}\left(1-t \cdot \frac{\tan \left(\theta_{k} / 2\right)+\tan \left(\theta_{k+1} / 2\right)}{\left|p_{k+1}-p_{k}\right|}\right)^{2} .
\end{aligned}
$$

Remark 5.3 This formula itself has already appeared in some papers aiming to find the discrete curvature at the vertices by using the offsets, see [2,4]. If we take the offset of the edges, then we have the following (at least) three possibilities. By computing the length of dotted curves in the figure, we can write the total length of each offset as follows:

$$
\begin{aligned}
& \text { Length } \Gamma_{h, t}^{(1)}=\text { Length } \Gamma_{h}-t \sum_{k} 2 \sin \frac{\theta_{k}}{2}, \quad \text { Length } \Gamma_{h, t}^{(2)}=\text { Length } \Gamma_{h}-t \sum_{k} \theta_{k}, \\
& \text { Length } \Gamma_{h, t}^{(3)}=\text { Length } \Gamma_{h}-t \sum_{k} 2 \tan \frac{\theta_{k}}{2} .
\end{aligned}
$$

Note the signature of the angles. The second curve $\Gamma_{h}^{(2)}$ is nothing but the normal cone method (or the boundary of the Minkowski sum with the disk) known in convex geometry. However, the only possible way to keep the number of the vertices unchanged during the offset procedure is the third one, and modifying the third formula gives our Steiner-type formula:

$$
\begin{aligned}
\text { Length } \Gamma_{h, t}^{(3)} & =\sum_{k}\left(l_{k}-2 t \tan \frac{\theta_{k}}{2}\right) \\
& =\sum_{k}\left(l_{k}-t \tan \frac{\theta_{k}}{2}+t \tan \frac{\theta_{k+1}}{2}\right)=\sum_{k} l_{k}\left(1-t \kappa\left(e_{k}\right)\right),
\end{aligned}
$$

where we put $\kappa\left(e_{k}\right)=\left(\tan \left(\theta_{k} / 2\right)+\tan \left(\theta_{k+1} / 2\right)\right) / l_{k}$.

Remark 5.4 (discrete Frenet-Serretformula) In Sect. 2, we remarked that the relation

$$
\tilde{N}_{k}=\frac{1}{L_{k}}\left(t_{k}-t_{k-1}\right), \quad t_{k}=\frac{p_{k+1}-p_{k}}{\left|p_{k+1}-p_{k}\right|}
$$

can be considered as a part of the Frenet-Serret formula. On the other hand, by some calculation we have

$$
\frac{1}{\left|p_{k+1}-p_{k}\right|}\left(N_{k+1}-N_{k}\right)=-\frac{\tan \left(\theta_{k} / 2\right)+\tan \left(\theta_{k+1} / 2\right)}{\left|p_{k+1}-p_{k}\right|} t_{k}=-\kappa\left(e_{k}\right) t_{k} .
$$

This formula can be seen as the other part of the Frenet-Serret formula. Note that the former formula is a formula on the vertex $p_{k}$ while the latter formula is a formula on the edge $\left[p_{k}, p_{k+1}\right]$. 


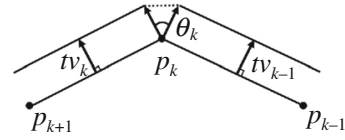

(a) $\Gamma_{h, t}^{(1)}$, connect by a segment

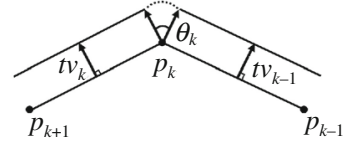

(b) $\Gamma_{h, t}^{(2)}$, connect by an arc

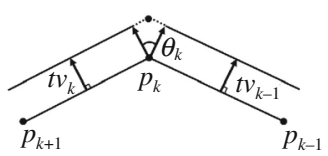

(c) $\Gamma_{h, t}^{(3)}$, connect by a wedge

Fig. 3 Three possibilities for constructing a new curve

\section{The Second Variation Formula}

In this section we consider the second variation formula of the length functional. We will follow the argument developed in [11].

Let $\Gamma_{h}=\left\{p_{k}\right\}_{k}$ be an equilibrium closed curve for the functional $L+\kappa$. Area. We say a variation is admissible (or permissible) if the variation is volume-preserving and fixes the boundary. We recall the first variation formula of the length and the 2-dimensional volume:

$$
\begin{aligned}
\frac{d}{d t} L & =\sum_{k}\left\langle\nabla_{p_{k}} L, p_{k}^{\prime}\right\rangle, & \nabla_{p_{k}} L & =R\left(v_{k}-v_{k-1}\right), \\
\frac{d}{d t} \text { Area } & =\sum_{k}\left\langle\nabla_{p_{k}} \text { Area, } p_{k}^{\prime}\right\rangle, & \nabla_{p_{k}} \text { Area } & =\frac{1}{2} R\left(p_{k+1}-p_{k-1}\right) .
\end{aligned}
$$

Note that if the variation is admissible, then we have

$$
0=\delta^{2} \text { Area }=\sum_{k}\left(\left\langle\delta \nabla_{p_{k}} \text { Area, } \delta p_{k}\right\rangle+\left\langle\nabla_{p_{k}} \text { Area, } \delta^{2} p_{k}\right\rangle\right)
$$

Lemma 6.1 Let $\Gamma_{h}=\left\{p_{k}\right\}_{k}$ be an equilibrium closed curve for $L+\kappa$. Area and $p_{k}(t)=p_{k}+t v_{k}+O\left(t^{2}\right)$ be an admissible variation. Then we have

$$
\delta^{2} L:=\left.\frac{d^{2} L}{d t^{2}}\right|_{t=0}=\sum_{k}\left\langle\delta\left(\nabla_{p_{k}} L+\kappa \nabla_{p_{k}} \text { Area }\right), v_{k}\right\rangle .
$$

Definition 6.2 (stability of discrete curves) Let $\Gamma_{h}=\left\{p_{k}\right\}_{k}$ be a closed equilibrium curve for the functional $L+\kappa$. Area. Then $\Gamma_{h}$ is said to be stable if $\delta^{2} L \geq 0$ for any admissible variation.

We introduce the matrices $Q^{L}$ and $Q^{A}$ as follows:

$$
{ }^{t} \vec{v} Q^{L} \vec{v}=\sum_{k}\left\langle\delta \nabla_{p_{k}} L, v_{k}\right\rangle, \quad{ }^{t} \vec{v} Q^{A} \vec{v}=\sum_{k}\left\langle\delta \nabla_{p_{k}} \text { Area, } v_{k}\right\rangle
$$

Then we can write $\delta^{2} L={ }^{t} \vec{v}\left(Q^{L}+\kappa Q^{A}\right) \vec{v}$.

Lemma $6.3{ }^{t} \vec{v} Q^{A} \vec{v}=\sum_{k}\left\langle v_{k}, R v_{k+1}\right\rangle$. 
Proof. The proof follows from direct computation:

$$
\begin{aligned}
{ }^{t} \vec{v} Q^{A} \vec{v} & =\sum_{k}\left\langle\delta \nabla_{p_{k}} \text { Area, } v_{k}\right\rangle=\frac{1}{2} \sum_{k}\left\langle\delta R\left(p_{k+1}-p_{k-1}\right), v_{k}\right\rangle \\
& =\frac{1}{2} \sum_{k}\left\langle R\left(v_{k+1}-v_{k-1}\right), v_{k}\right\rangle=\sum_{k}\left\langle v_{k}, R v_{k+1}\right\rangle .
\end{aligned}
$$

Proposition 6.4 (second variation formula for the length functional)

$$
Q^{L}=\sum_{k} \frac{1}{l_{k}}\left(\left|v_{k+1}-v_{k}\right|^{2}-\left\langle v_{k+1}-v_{k}, R v_{k}\right\rangle^{2}\right)=\sum_{k}\left(\left|\nabla v_{k}\right|^{2}-\left\langle\nabla v_{k}, R v_{k}\right\rangle^{2}\right) l_{k},
$$

and therefore we have the following second variation formula for the length functional:

$$
\delta^{2} L=\sum_{k}\left(\left(\left|\nabla v_{k}\right|^{2}-\left\langle\nabla v_{k}, R v_{k}\right\rangle^{2}\right) l_{k}+\kappa\left\langle v_{k}, R v_{k+1}\right\rangle\right)
$$

Proof. By using the fact that $l_{k} \delta v_{k}=R\left(v_{k+1}-v_{k}\right)-\left\langle R\left(v_{k+1}-v_{k}\right), v_{k}\right\rangle v_{k}$, we have

$$
\begin{aligned}
Q^{L} & =\sum_{k}\left\langle\delta v_{k}, R\left(v_{k+1}-v_{k}\right)\right\rangle=\sum_{k}\left\langle R\left(\nabla v_{k}\right)-\left\langle R\left(\nabla v_{k}\right), v_{k}\right\rangle v_{k}, R\left(\nabla v_{k}\right)\right\rangle l_{k} \\
& =\sum_{k}\left(\left|\nabla v_{k}\right|^{2}-\left\langle R\left(\nabla v_{k}\right), v_{k}\right\rangle^{2}\right) l_{k}=\sum_{k}\left(\left|\nabla v_{k}\right|^{2}-\left\langle\nabla v_{k}, R v_{k}\right\rangle^{2}\right) l_{k} .
\end{aligned}
$$

In the section on the Steiner-type formula, we used the vector

$$
N_{k}:=\frac{R\left(v_{k-1}-v_{k}\right)}{\sin \theta_{k}}=\frac{1}{1+\cos \theta_{k}}\left(v_{k}+v_{k-1}\right)
$$

as the "normal vector" at the vertex $p_{k}$. If we define the "tangent vector" $T_{k}$ as $T_{k}=$ $-R N_{k}$, then we can decompose the variation vector $v_{k}$ as

$$
v_{k}=\psi_{k} N_{k}+\eta_{k} T_{k}=\psi_{k} N_{k}-\eta_{k} R N_{k}
$$

where $\psi, \eta: V \rightarrow \mathbb{R}$ are some functions on the vertices. If $\eta_{k}=0$ for all $k$, we call the variation the normal variation. In the following we will use this notation.

Lemma 6.5 The first variation formula of the volume can be written as

$$
\delta \text { Area }=\frac{1}{2} \sum_{k}\left(\psi_{k}\left(l_{k}+l_{k-1}\right)+\eta_{k}\left(l_{k}-l_{k-1}\right) \tan \frac{\theta_{k}}{2}\right) .
$$

In particular, if the curve $\Gamma_{h}=\left\{p_{k}\right\}_{k}$ satisfies $l_{k} \equiv l_{0}$, then a variation $p_{k}(t)=$ $p_{k}+t\left(\psi_{k} N_{k}+\eta_{k} T_{k}\right)+O\left(t^{2}\right)$ is volume-preserving if $\sum_{k} \psi_{k}=0$. 
Proof. Using the first variation formula, we have

$$
\begin{aligned}
\delta \text { Area }= & \frac{1}{2} \sum_{k}\left\langle R\left(p_{k+1}-p_{k-1}\right), v_{k}\right\rangle=\frac{1}{2} \sum_{k}\left\langle R\left(p_{k+1}-p_{k-1}\right), \psi_{k} N_{k}+\eta_{k} T_{k}\right\rangle \\
= & \frac{1}{2} \sum_{k}\left[\psi_{k} l_{k} \frac{\left\langle v_{k}, R v_{k-1}\right\rangle}{\sin \theta_{k}}+\psi_{k} l_{k-1} \frac{\left\langle v_{k-1},-R v_{k}\right\rangle}{\sin \theta_{k}}\right. \\
& \left.+\frac{\eta_{k}}{\sin \theta_{k}}\left(l_{k}-l_{k-1}\right)\left(1-\cos \theta_{k}\right)\right] \\
= & \frac{1}{2} \sum_{k}\left(\psi_{k}\left(l_{k}+l_{k-1}\right)+\eta_{k}\left(l_{k}-l_{k-1}\right) \tan \frac{\theta_{k}}{2}\right) .
\end{aligned}
$$

Remark 6.6 For functions $\psi_{k}$ satisfying $\sum_{k} \psi_{k}=0$, we can find variations whose variation vector fields are $\psi_{k} N_{k}$. The proof is completely the same as in [1].

Recall that if $\left\{p_{k}\right\}_{k}$ is an equilibrium curve of the functional $L+\kappa \cdot$ Area, then we have $l_{k} \equiv l_{0}, \theta_{k} \equiv \theta_{0}$, and $\kappa l_{0}=2 \tan \left(\theta_{0} / 2\right)$.

\section{Lemma 6.7}

$$
\left|v_{k+1}-v_{k}\right|^{2}-\left\langle v_{k+1}-v_{k}, R v_{k}\right\rangle^{2}=\left[\left(\psi_{k+1}-\psi_{k}\right)+\left(\eta_{k+1}+\eta_{k}\right) \tan \frac{\theta_{0}}{2}\right]^{2} .
$$

Therefore we have

$$
\left|\nabla v_{k}\right|^{2}-\left\langle\nabla v_{k}, R v_{k}\right\rangle^{2}=\left(\nabla \psi_{k}+\frac{\kappa}{2}\left(\eta_{k}+\eta_{k+1}\right)\right)^{2}
$$

Proof. Recall that

$$
\begin{aligned}
\left\langle N_{k},-R N_{k+1}\right\rangle & =\tan \frac{\theta_{k}}{2}+\tan \frac{\theta_{k+1}}{2}=2 \tan \frac{\theta_{0}}{2}, \\
\left\langle N_{k}, N_{k+1}\right\rangle & =1-\tan \frac{\theta_{k}}{2} \tan \frac{\theta_{k+1}}{2}=1-\tan ^{2} \frac{\theta_{0}}{2} .
\end{aligned}
$$

If we note that $\left|N_{k}\right|=1 / \cos \left(\theta_{0} / 2\right)$, then

$$
\begin{aligned}
\mid v_{k+1} & -\left.v_{k}\right|^{2} \\
= & \left|\psi_{k+1} N_{k+1}-\psi_{k} N_{k}\right|^{2}+2\left\langle\psi_{k+1} N_{k+1}-\psi_{k} N_{k}, \eta_{k+1} T_{k+1}-\eta_{k} T_{k}\right\rangle \\
& +\left|\eta_{k+1} T_{k+1}-\eta_{k} T_{k}\right|^{2} \\
= & \frac{\psi_{k+1}^{2}}{\cos ^{2}\left(\theta_{0} / 2\right)}-2 \psi_{k} \psi_{k+1}\left(1-\tan ^{2} \frac{\theta_{0}}{2}\right)+\frac{\psi_{k}^{2}}{\cos ^{2}\left(\theta_{0} / 2\right)} \\
& -2\left\langle N_{k}, R N_{k+1}\right\rangle\left(\psi_{k+1} \eta_{k}-\psi_{k} \eta_{k+1}\right) \\
& +\frac{\eta_{k+1}^{2}}{\cos ^{2}\left(\theta_{0} / 2\right)}-2 \eta_{k} \eta_{k+1}\left(1-\tan ^{2} \frac{\theta_{0}}{2}\right)+\frac{\eta_{k}^{2}}{\cos ^{2}\left(\theta_{0} / 2\right)}
\end{aligned}
$$




$$
\begin{aligned}
= & \left(\psi_{k+1}^{2}+\psi_{k}^{2}+\eta_{k+1}^{2}+\eta_{k}^{2}\right)\left(1+\tan ^{2} \frac{\theta_{0}}{2}\right) \\
& -2\left(\psi_{k} \psi_{k+1}+\eta_{k} \eta_{k+1}\right)\left(1-\tan ^{2} \frac{\theta_{0}}{2}\right)+4\left(\eta_{k} \psi_{k+1}-\eta_{k+1} \psi_{k}\right) \tan \frac{\theta_{0}}{2} .
\end{aligned}
$$

Similarly, since

$$
\begin{aligned}
\left\langle v_{k+1}-v_{k}, R v_{k}\right\rangle & =\left\langle\psi_{k+1} N_{k+1}-\psi_{k} N_{k}+\eta_{k+1} T_{k+1}-\eta_{k} T_{k}, R v_{k}\right\rangle \\
= & \psi_{k+1} \frac{\left\langle v_{k}-v_{k+1}, v_{k}\right\rangle}{\sin \theta_{k+1}}-\psi_{k} \frac{\left\langle v_{k-1}-v_{k}, v_{k}\right\rangle}{\sin \theta_{k}} \\
& -\eta_{k+1} \frac{\left\langle v_{k+1}-v_{k}, R v_{k}\right\rangle}{\sin \theta_{k+1}}+\eta_{k} \frac{\left\langle v_{k}-v_{k-1}, R v_{k}\right\rangle}{\sin \theta_{k}} \\
= & \left(\psi_{k+1}+\psi_{k}\right) \tan \frac{\theta_{0}}{2}-\left(\eta_{k+1}-\eta_{k}\right),
\end{aligned}
$$

we have

$$
\begin{aligned}
\left\langle v_{k+1}-v_{k}, R v_{k}\right\rangle^{2}= & \left(\psi_{k+1}+\psi_{k}\right)^{2} \tan ^{2} \frac{\theta_{0}}{2} \\
& -2\left(\psi_{k+1}+\psi_{k}\right)\left(\eta_{k+1}-\eta_{k}\right) \tan \frac{\theta_{0}}{2}+\left(\eta_{k+1}-\eta_{k}\right)^{2} .
\end{aligned}
$$

Subtracting these factors we have

$$
\begin{aligned}
\mid v_{k+1} & -\left.v_{k}\right|^{2}-\left\langle v_{k+1}-v_{k}, R v_{k}\right\rangle^{2} \\
= & \psi_{k+1}^{2}+\psi_{k}^{2}+\left(\eta_{k+1}^{2}+\eta_{k}^{2}\right) \tan ^{2} \frac{\theta_{0}}{2}-2 \psi_{k} \psi_{k+1} \tan ^{2} \frac{\theta_{0}}{2}+2 \eta_{k} \eta_{k+1} \\
& +2\left(2 \eta_{k} \psi_{k+1}-2 \eta_{k+1} \psi_{k}+\psi_{k+1} \eta_{k+1}-\psi_{k+1} \eta_{k}+\psi_{k} \eta_{k+1}-\psi_{k} \eta_{k}\right) \tan \frac{\theta_{0}}{2} \\
& -2\left(\psi_{k} \psi_{k+1}+\eta_{k} \eta_{k+1}\right)+2\left(\psi_{k} \psi_{k+1}+\eta_{k} \eta_{k+1}\right) \tan ^{2} \frac{\theta_{0}}{2} \\
= & \left(\psi_{k+1}-\psi_{k}\right)^{2}+\left(\eta_{k+1}+\eta_{k}\right)^{2} \tan ^{2} \frac{\theta_{0}}{2}+2\left(\psi_{k+1}-\psi_{k}\right)\left(\eta_{k+1}+\eta_{k}\right) \tan \frac{\theta_{0}}{2} \\
\quad= & {\left[\left(\psi_{k+1}-\psi_{k}\right)+\left(\eta_{k+1}+\eta_{k}\right) \tan \frac{\theta_{0}}{2}\right]^{2} . }
\end{aligned}
$$

\section{Lemma 6.8}

$\left\langle v_{k}, R v_{k+1}\right\rangle=-2\left(\psi_{k} \psi_{k+1}+\eta_{k} \eta_{k+1}\right) \tan \frac{\theta_{0}}{2}-\left(\eta_{k} \psi_{k+1}-\eta_{k+1} \psi_{k}\right)\left(1-\tan ^{2} \frac{\theta_{0}}{2}\right)$.

Proof. This is also a simple calculation:

$\left\langle v_{k}, R v_{k+1}\right\rangle=\left\langle\psi_{k} N_{k}-\eta_{k} R N_{k}, \psi_{k+1} R N_{k+1}+\eta_{k+1} N_{k+1}\right\rangle$ 


$$
\begin{aligned}
& =\left(\psi_{k} \psi_{k+1}+\eta_{k} \eta_{k+1}\right)\left\langle N_{k}, R N_{k+1}\right\rangle-\left(\eta_{k} \psi_{k+1}-\eta_{k+1} \psi_{k}\right)\left\langle N_{k}, N_{k+1}\right\rangle \\
& =-2\left(\psi_{k} \psi_{k+1}+\eta_{k} \eta_{k+1}\right) \tan \frac{\theta_{0}}{2}-\left(\eta_{k} \psi_{k+1}-\eta_{k+1} \psi_{k}\right)\left(1-\tan ^{2} \frac{\theta_{0}}{2}\right) .
\end{aligned}
$$

Theorem 6.9 (second variation formula for the length functional)

$$
\delta^{2} L=\sum_{k}\left[\left|\nabla \psi_{k}\right|^{2}-\kappa^{2} \psi_{k} \psi_{k+1}+\left(\kappa \nabla \psi_{k}\left(\eta_{k+1}+\eta_{k}\right)+\left|\nabla \eta_{k}\right|^{2}\right) \tan ^{2} \frac{\theta_{0}}{2}\right] l_{0} .
$$

In particular, for the normal variation we have

$$
\delta^{2} L=\sum_{k}\left(\left|\nabla \psi_{k}\right|^{2}-\kappa^{2} \psi_{k} \psi_{k+1}\right) l_{0}=-\sum_{k} \psi_{k}\left(\Delta \psi_{k}+\kappa^{2} \psi_{k+1}\right) l_{0}
$$

where we use the integration by parts and take the line element at the vertex as $L_{k}=\left(l_{k}+l_{k-1}\right) / 2=l_{0}$.

Proof By using the previous lemmas, we have

$$
\begin{aligned}
\left(\left|\nabla v_{k}\right|^{2}-\left\langle\nabla v_{k}, R v_{k}\right\rangle^{2}\right) l_{k}+\kappa\left\langle v_{k}, R v_{k+1}\right\rangle \\
=\left(\left|\nabla \psi_{k}\right|^{2}+\kappa \nabla \psi_{k}\left(\eta_{k+1}+\eta_{k}\right)+\frac{\kappa^{2}}{4}\left(\eta_{k+1}+\eta_{k}\right)^{2}\right) l_{0} \\
\quad-\kappa^{2} l_{0}\left(\psi_{k} \psi_{k+1}+\eta_{k} \eta_{k+1}\right)-\kappa\left(\eta_{k} \psi_{k+1}-\eta_{k+1} \psi_{k}\right)\left(1-\tan ^{2} \frac{\theta_{0}}{2}\right) \\
=\left|\nabla \psi_{k}\right|^{2} l_{0}+\kappa \nabla \psi_{k}\left(\eta_{k+1}+\eta_{k}\right) l_{0}+\frac{\kappa^{2}}{4}\left(\eta_{k+1}-\eta_{k}\right)^{2} l_{0}-\kappa^{2} \psi_{k} \psi_{k+1} l_{0} \\
\quad-\kappa\left(\left(\psi_{k+1}-\psi_{k}\right)\left(\eta_{k+1}+\eta_{k}\right)-\left(\psi_{k+1} \eta_{k+1}-\psi_{k} \eta_{k}\right)\right)\left(1-\tan ^{2} \frac{\theta_{2}}{2}\right) \\
=\left|\nabla \psi_{k}\right|^{2} l_{0}+\kappa \tan ^{2} \frac{\theta_{0}}{2} \cdot \nabla \psi_{k}\left(\eta_{k+1}+\eta_{k}\right) l_{0}+\frac{\kappa^{2}}{4}\left(\eta_{k+1}-\eta_{k}\right)^{2} l_{0}-\kappa^{2} \psi_{k} \psi_{k+1} l_{0} \\
\quad+\kappa\left(\psi_{k+1} \eta_{k+1}-\psi_{k} \eta_{k}\right)\left(1-\tan ^{2} \frac{\theta_{0}}{2}\right) .
\end{aligned}
$$

Summing up, we get the desired result.

\section{Instability of Non-Convex Regular Polygons}

In this section we will prove that non-convex regular polygons and convex regular polygons with multiplicity are unstable. To prove this, we find a special variation with a help of the following discrete version of Wirtinger's inequality: 
Theorem 7.1 (discrete Wirtinger's inequality [6]) Let $\psi_{0}, \ldots, \psi_{n}$ be $n+1$ real numbers such that

$$
\psi_{0}=\psi_{n}, \quad \sum_{k=0}^{n-1} \psi_{k}=0
$$

Then we have

$$
\sum_{k=0}^{n-1}\left(\psi_{k+1}-\psi_{k}\right)^{2} \geq 4 \sin ^{2} \frac{\pi}{n} \sum_{k=0}^{n-1} \psi_{k}^{2}
$$

and the equality holds if and only if there exist $A, B \in \mathbb{R}$ such that

$$
\psi_{k}=A \cos \frac{2 \pi k}{n}+B \sin \frac{2 \pi k}{n} .
$$

In the following, we will consider the normal variation, i.e., the variation of the form

$$
p_{k}(t)=p_{k}+t \psi_{k} N_{k}+O\left(t^{2}\right), \quad N_{k}=\frac{v_{k}+v_{k-1}}{1+\cos \theta_{0}},
$$

where $\psi_{k}$ satisfies $\sum_{k} \psi_{k}=0$. By the second variation formula (Theorem 6.9) we have

$\delta^{2} L=\sum_{k}\left(\left|\nabla \psi_{k}\right|^{2}-\kappa^{2} \psi_{k} \psi_{k+1}\right) l_{0}=\sum_{k} \frac{1}{l_{0}}\left[\left(\psi_{k+1}-\psi_{k}\right)^{2}-4 \psi_{k} \psi_{k+1} \tan ^{2} \frac{m \pi}{n}\right]$

for any admissible variations, where we use the relation $\kappa l_{0}=2 \tan \left(\theta_{0} / 2\right)$, put $\theta_{0}=$ $2 m \pi / n$ for some $m \in \mathbb{Z}$, and assume that $m / n \neq 1 / 2$.

Theorem 7.2 (instability of non-convex regular polygons) Let $n \geq 5$. By taking $\psi_{k}$ as in $(7.2),(A, B) \neq(0,0)$, we have

$$
\delta^{2} L=\frac{4}{l_{0}}\left[\sin ^{2} \frac{\pi}{n}-\cos \frac{2 \pi}{n} \tan ^{2} \frac{m \pi}{n}\right] \sum_{k} \psi_{k}^{2} .
$$

In particular, $\delta^{2} L<0$ for $2 \leq m \leq n-2$, i.e., non-convex regular polygons are unstable.

Proof By the discrete Wirtinger's inequality we have

$$
\delta^{2} L \geq \sum_{k} \frac{4}{l_{0}}\left(\psi_{k}^{2} \sin ^{2} \frac{\pi}{n}-\psi_{k} \psi_{k+1} \tan ^{2} \frac{m \pi}{n}\right)
$$


In the following we use (7.2) and put $\varphi_{k}=-A \sin (2 \pi k / n)+B \cos (2 \pi k / n)$. Then we have

$$
\psi_{k+1}=\psi_{k} \cos \frac{2 \pi}{n}+\varphi_{k} \sin \frac{2 \pi}{n}, \quad \psi_{k} \varphi_{k}=\frac{B^{2}-A^{2}}{2} \sin \frac{4 k \pi}{n}+A B \cos \frac{4 k \pi}{n}
$$

If we note that $\sum_{k} \psi_{k} \varphi_{k}=0$, then

$$
\begin{aligned}
\delta^{2} L & =\sum_{k} \frac{4}{l_{0}}\left[\psi_{k}^{2} \sin ^{2} \frac{\pi}{n}-\psi_{k}\left(\psi_{k} \cos \frac{2 \pi}{n}+\varphi_{k} \sin \frac{2 \pi}{n}\right) \tan ^{2} \frac{m \pi}{n}\right] \\
& =\frac{4}{l_{0}}\left[\sin ^{2} \frac{\pi}{n}-\cos \frac{2 \pi}{n} \tan ^{2} \frac{m \pi}{n}\right] \sum_{k} \psi_{k}^{2} .
\end{aligned}
$$

If $m=1$ or $m=n-1$, then

$$
\delta^{2} L \geq \frac{4}{l_{0}} \sin ^{2} \frac{\pi}{n} \tan ^{2} \frac{\pi}{n} \sum_{k} \psi_{k}^{2} \geq 0
$$

On the other hand, for $2 \leq m \leq n-2$ and $(A, B) \neq(0,0)$ we have

$$
\delta^{2} L \leq-\frac{4 \sin ^{2}(\pi / n)\left(1+2 \cos ^{2}(\pi / n)\right)}{l_{0} \cos (2 \pi / n)} \sum_{k} \psi_{k}^{2}<0
$$

where we use the fact $\tan ^{2}(m \pi / n) \geq \tan ^{2}(2 \pi / n)$ if $2 \leq m \leq n-2$, and $\cos (2 \pi / n)>0$ if $n \geq 5$. This proves the statement.

\section{Concluding Remarks}

We observe the second variation formula from the analysis of the Jacobi operator. We can modify the equation (7.1) as follows:

$$
\delta^{2} L=\frac{1}{l_{0}} \sum_{k}\left(-\alpha \psi_{k-1}+2 \psi_{k}-\alpha \psi_{k+1}\right) \psi_{k}=\frac{\langle H \Psi, \Psi\rangle}{l_{0}},
$$

where we put $\alpha=1+2 \tan ^{2}(m \pi / n)$ and

$$
H=\left(\begin{array}{cccccc}
2 & -\alpha & 0 & \cdots & 0 & -\alpha \\
-\alpha & 2 & -\alpha & \cdots & 0 & 0 \\
0 & -\alpha & 2 & \cdots & 0 & 0 \\
\vdots & \vdots & \vdots & \ddots & \vdots & \vdots \\
0 & 0 & 0 & \cdots & 2 & -\alpha \\
-\alpha & 0 & 0 & \cdots & -\alpha & 2
\end{array}\right), \quad \Psi=\left(\begin{array}{c}
\psi_{1} \\
\psi_{2} \\
\psi_{3} \\
\vdots \\
\psi_{n}
\end{array}\right)
$$


Since $H$ is a circulant matrix, the eigenvalues $\lambda_{j}$ can be calculated explicitly, see e.g. [5]:

$$
\lambda_{j}=2-2 \alpha \cos \frac{2 \pi j}{n}=\frac{4 \cos ^{2}(j \pi / n)}{\cos ^{2}(m \pi / n)}\left(\tan ^{2} \frac{j \pi}{n}-\sin ^{2} \frac{m \pi}{n}\right), \quad j=1, \ldots, n .
$$

The corresponding eigenvectors are

$$
e_{j}={ }^{t}\left(1, \omega^{j}, \ldots, \omega^{j(n-1)}\right) \in \mathbb{C}^{n}, \quad \omega=\exp \frac{2 \pi \sqrt{-1}}{n} .
$$

The condition $\sum_{k} \psi_{k}=0$ is equivalent to the condition that $\Psi={ }^{t}\left(\psi_{1}, \ldots, \psi_{n}\right)$ is perpendicular to $e_{n}={ }^{t}(1, \ldots, 1)$. Therefore we only consider the eigenvalues $\lambda_{1}, \ldots, \lambda_{n-1}$.

Lemma 8.1 For $m \leq j \leq n-m, \lambda_{j}>0$. In particular, $\lambda_{j}>0$ for $1 \leq j \leq n-1$ if $m=1$, i.e., the convex regular polygon case.

Proof. This is a direct calculation:

$$
\tan ^{2} \frac{j \pi}{n}-\sin ^{2} \frac{m \pi}{n} \geq \tan ^{2} \frac{m \pi}{n}-\sin ^{2} \frac{m \pi}{n}=\sin ^{2} \frac{m \pi}{n} \tan ^{2} \frac{m \pi}{n}>0 .
$$

Theorem 8.2 Assume $m \geq 2$. For $1 \leq j \leq m / 2$ or $n-m / 2 \leq j \leq n-1$, we have $\lambda_{j}<0$. Therefore, the index of a non-convex regular polygon or a convex polygon with multiplicity is at least $\lfloor m / 2\rfloor$.

Proof. Under the above conditions, we have

$$
\tan ^{2} \frac{j \pi}{n}-\sin ^{2} \frac{m \pi}{n} \leq \tan ^{2} \frac{j \pi}{n}-\sin ^{2} \frac{2 j \pi}{n}<0 .
$$

Remark 8.3 More precisely, $\lambda_{j}<0$ for $j<(n / \pi) \arctan (\sin (m \pi / n))$.

Remark 8.4 From another point of view, a non-convex regular polygon is the highfrequency component of the discrete Fourier expansion of the polygon. More precisely, any polygon in $\mathbb{R}^{2}$ with $n$ vertices can be regarded as a point in $\mathbb{R}^{2 n} \simeq \mathbb{C}^{n}$. Each eigenvector $e_{k}$ in (8.1) corresponds to the regular $n$-gon and $\left\{e_{1}, \ldots, e_{n}\right\}$ forms a basis of $\mathbb{C}^{n}$. Therefore, any polygon in $\mathbb{R}^{2}$ can be written as a linear combination of regular $n$-gons and this fact corresponds to the discrete Fourier expansion.

Acknowledgements The author would like to express his gratitude to Professor Miyuki Koiso and Professor Hisashi Naito for invaluable comments and fruitful discussions. This work is partially supported by JST CREST Grant Number JPMJCR1911, Japan.

Open Access This article is licensed under a Creative Commons Attribution 4.0 International License, which permits use, sharing, adaptation, distribution and reproduction in any medium or format, as long as you give appropriate credit to the original author(s) and the source, provide a link to the Creative Commons licence, and indicate if changes were made. The images or other third party material in this article are included in the article's Creative Commons licence, unless indicated otherwise in a credit line to the material. If 
material is not included in the article's Creative Commons licence and your intended use is not permitted by statutory regulation or exceeds the permitted use, you will need to obtain permission directly from the copyright holder. To view a copy of this licence, visit http://creativecommons.org/licenses/by/4.0/.

\section{References}

1. Barbosa, J.L., do Carmo, M.: Stability of hypersurfaces with constant mean curvature. Math. Z. 185(3), 339-353 (1984)

2. Bauer, U., Polthier, K., Wardetzky, M.: Uniform convergence of discrete curvatures from nets of curvature lines. Discrete Comput. Geom. 43(4), 798-823 (2010)

3. Chow, B., Glickenstein, D.: Semidiscrete geometric flows of polygons. Am. Math. Mon. 114(4), 316328 (2007)

4. Crane, K., Wardetzky, M.: A glimpse into discrete differential geometry. Not. Am. Math. Soc. 64(10), 1153-1159 (2017)

5. Davis, P.J.: Circulant Matrices. A Wiley-Interscience Publication. Pure and Applied Mathematics. Wiley, New York (1979)

6. Fan, K., Taussky, O., Todd, J.: Discrete analogs of inequalities of Wirtinger. Monatsh. Math. 59, 73-90 (1955)

7. Hatakeyama, Y.: Some Attempts to Construct Geometric Theories to Represent and Explicate Physical Phenomena. PhD thesis, Kyushu University (2019)

8. Hoffmann, T.: Discrete Differential Geometry of Curves and Surfaces. COE Lecture Note, vol. 18. Math-for-Industry (MI) Lecture Note Series. Kyushu University, Faculty of Mathematics, Fukuoka (2009)

9. Pinkall, U., Polthier, K.: Computing discrete minimal surfaces and their conjugates. Exp. Math. 2(1), 15-36 (1993)

10. Polthier, K.: Polyhedral Surfaces with Constant Mean Curvature. Habilitation thesis, Technische Universität Berlin (2002)

11. Polthier, K., Rossman, W.: Discrete constant mean curvature surfaces and their index. J. Reine Angew. Math. 549, 47-77 (2002)

12. Wardetzky, M., Mathur, S., Kälberer, F., Grinspun, E.: Discrete Laplace operators: no free lunch. In: Eurographics Symposium on Geometry Processing (Barcelona 2007), pp. 33-37. Eurographics Association, Goslar (2007)

Publisher's Note Springer Nature remains neutral with regard to jurisdictional claims in published maps and institutional affiliations. 